\title{
Application of Chaotic Motion to Industrial Compactors
}

\author{
K. T. Chau, Zheng Wang \\ Department of Electrical and Electronic Engineering, The University of Hong Kong, Hong Kong, China
}

\begin{abstract}
This paper proposes and implements a novel electrically chaotic compactor. A modified time-delay feedback control method is applied to chaoize a DC motor in the compactor. The Poincaré map, the bifurcation diagram and the maximum Lyapunov exponent are used to analyze the chaos in the electrically chaotic compactor. Simulation comparison not only proves that the electrically chaotic compactor offers better compacting performance than the constant speed compactor but also verifies that the electrically chaotic compactor offers the advantages of good controllability and high flexibility rather than the mechanically chaotic compactor.
\end{abstract}

\section{INTRODUCTION}

Vibratory compaction is an important industrial process around the world. A vibratory compactor functions to effectively compact soil and concrete materials, hence achieving the desired density. With the rapid development of construction, there is an urgent need for innovation in the area of vibratory compaction. Reference [1] represents a mechanical analogy for the soil compactor system in terms of the proposed two-degree-of-freedom system. In this research the dynamic interaction between the frame and the drum is described with suspension stiffness $K_{r}$ and suspension damping $C_{f}$ while the dynamic interaction between the drum and the soil is described with soil stiffness $K_{r}$ and soil damping $C_{s}$. It is proved that a heavier frame and lighter drum will impart more compaction effort to the soil for the same static weight in their research. Reference [2] researches on improving the vibratory compaction effect by mounting the vibratory drum on the centrifugal, vertical and horizontal position of the compactor respectively. The vertical vibratory compactor is revealed to have the best compaction performance at low frequency. Different from the research on these periodic motion, Reference [3] proposes an innovate compactor to improve compaction effect with chaotic motion. This new compactor termed mechanically chaotic compactor is constructed by rotating three interlinked eccentric masses at a constant speed. The experiment result shows that this compactor exhibits better compaction performance than the conventional constant speed compactor because of its wider amplitude of displacement and wider power spectral density distribution.

The purpose of this paper is to propose and implement a novel electrically chaotic compactor. Different from the mechanically chaotic one, this electrically chaotic compactor will adopt time-delay feedback control to chaoize a DC motor which is coupled with an eccentric mass, hence offering the advantages of good controllability and high flexibility.
In Section II, dynamic equations of the constant speed compactor and the electrically chaotic compactor will be provided, and in Section III analysis about chaos in the electrically chaotic compactor is presented. Then in Section IV, Simulation comparison not only proves that the electrically chaotic compactor offers better compaction performance than the constant speed compactor but also verifies that the electrically chaotic compactor offers the advantages of good controllability and high flexibility rather than the mechanically chaotic compactor. Finally, in Section V conclusions will be drawn.

\section{SYSTEM MODELING}

Fig. 1 shows the structure of constant speed compactor and electrically chaotic compactor, where $M_{f}, M_{p}, M_{c}$ are the weights of the framework, the vibratory plate and the eccentric mass respectively. $K_{f}$ is suspension stiffness and $C_{f}$ is suspension damping. Here the soil model is presented by soil stiffness $K_{s}$ and soil damping $C_{s}$ [1].

The dynamic equations of constant speed compactor are given by:

$$
\begin{aligned}
& M_{f} \ddot{y}_{f}=-K_{f}\left(y_{f}-y_{p}\right)-C_{f}\left(\dot{y}_{f}-\dot{y}_{p}\right)+M_{f} g \\
& \left(M_{p}+M_{c}\right) \ddot{y}_{p}-M_{c} \omega^{2} r \cos \omega t=-K_{s} y_{p}-C_{s} \dot{y}_{p} \\
& +K_{f}\left(y_{f}-y_{p}\right)+C_{f}\left(\dot{y}_{f}-\dot{y}_{p}\right)+\left(M_{p}+M_{c}\right) g
\end{aligned}
$$

where $r$ is the gravitational eccentric length of the eccentric mass.

The eccentric mass in the innovated electrically chaotic compactor is driven by a DC motor. The modified time-delay feedback control system is used to chaoize the

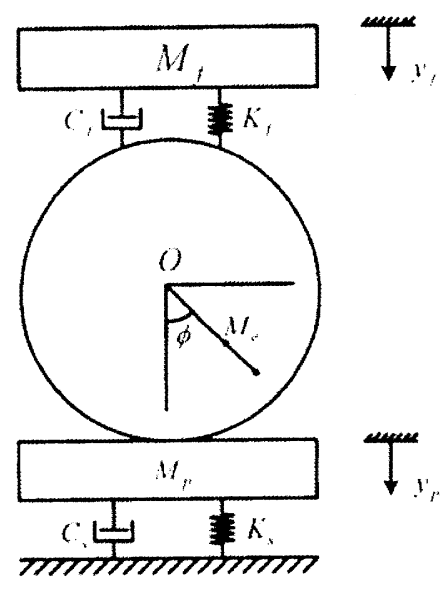

Fig. 1. Structure of compactor system. 


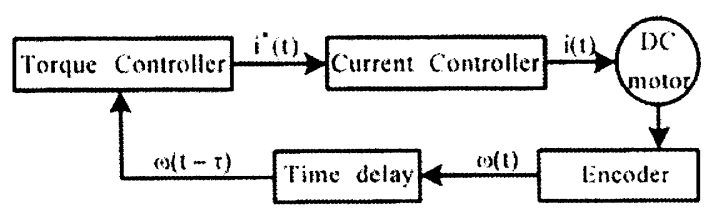

Fig. 2. Time-delay feedback control system.

DC motor. Different from the control system used in chaotic mixing [4], the modified control system can chaoize the DC motor with large inertia besides small one. Fig. 2 shows the block diagram of modified time-delay feedback control system. The corresponding control system can be expressed as:

$$
\begin{aligned}
& \frac{d \omega}{d t}=-\frac{B+k J}{J} \omega(t)+\frac{\mu \xi(B+k J)}{J} \sin \left[\frac{\omega(t-\tau)}{\xi}\right]-\frac{T_{1}}{J} \\
& \frac{d \phi}{d t}=\omega
\end{aligned}
$$

where $B$ is the viscous damping coefficient of DC motor, $J$ is the rotating inertia of the compactor system, $T_{1}$ is the load torque, $\tau$ is the time-delay parameter, $\mu$ is the torque parameter, $\xi$ is the speed parameter and $k$ is the control gain. Combined (3-4) with (1-2), the dynamic equations of the electrically chaotic compactor can be formed.

In our research, the parameters of constant speed compactor and electrically chaotic compactor are listed in TABLE I. $B_{w}$ is the width of plate and $L$ is the length of plate. Soil stiffness $K_{s}$ and soil damping $C_{s}$ can be computed with (5-6):

$$
\begin{aligned}
& r_{0}=\sqrt{\frac{B_{w} L}{\pi}} \\
& K_{s}=\frac{4 G r_{0}}{1-v}
\end{aligned}
$$

Soil damping can be computed with (7-8) [5]:

TABLE I

PARAMETERS OF COMPACTOR SYSTEM

\begin{tabular}{lll}
\hline Parameter & Value & Unit \\
\hline$M_{f}$ & 50 & $\mathrm{~kg}$ \\
$M_{p}$ & 50 & $\mathrm{~kg}$ \\
$M_{e}$ & 50 & $\mathrm{~kg}$ \\
$r$ & 0.15 & $\mathrm{~m}$ \\
$K_{f}$ & $5.25 \times 10^{6}$ & $\mathrm{~N} / \mathrm{m}$ \\
$C_{f}$ & $2.625 \times 10^{3}$ & $\mathrm{Ns} / \mathrm{m}$ \\
$B_{w}$ & 0.2 & $\mathrm{~m}$ \\
$L$ & 0.4 & $\mathrm{~m}$ \\
\hline
\end{tabular}

TABLE II

PARAMETERS OF SOIL

\begin{tabular}{lll}
\hline Soil type & $\mathrm{G}(\mathrm{Pa})$ & $v$ \\
\hline Soft clay & $6.6 \times 10^{6}$ & 0.16 \\
Medium clay & $1.6 \times 10^{7}$ & 0.16 \\
Stiff clay & $8.3 \times 10^{7}$ & 0.16 \\
\hline
\end{tabular}

$B_{z}=\frac{(1-v)\left(M_{c}+M_{p}\right)}{4 \rho r_{0}^{3}}$

$D_{z}=\frac{0.425}{\sqrt{B_{z}}}$

$C_{s}=2 D \sqrt{M_{e}+M_{p}}$

The natural frequency of soil can be computed with (10) [2], [5]:

$f_{n}=\frac{\sqrt{K_{s} /\left(M_{e}+M_{p}\right)}}{2 \pi}$

The parameters of three different clays: soft clay, medium clay and stiff clay and listed in TABLE II [6].

\section{ChaOtic ANALYSIS .}

Let $X(t)=\omega(t)$. The sampling time is set as $\tau$, and the Poincaré map that maps a sample $X_{n}$ of $X(t)$ at $t=n \tau$ to its successive one $X_{n+1}$ at $t=(n+1) \tau$ is defined as $P: \mathfrak{R} \rightarrow \mathfrak{R}, X_{n+1}=P\left(X_{n}\right)$.

The dynamic equations of the DC motor can be expressed with the following equations [4]:

$\frac{d \omega(t)}{d t}=-\frac{B}{J} \omega(t)+\frac{K_{T}}{J} i(t)-\frac{T_{t}}{J}$

$T_{e}=K_{r} i(t)$

where $T_{e}$ is the motor torque and $K_{T}$ is the torque constant. In this paper the feedback control law is chosen as:

$T_{e}=\mu \xi(B+k J) \sin \left[\frac{\omega(t-\tau)}{\xi}\right]-k J \omega(t)$

So the systems dynamics can be written as:

$\frac{d \omega(t)}{d t}=-\frac{B+k J}{J} \omega(t)+\frac{\mu \xi(B+k J)}{J} \sin \left[\frac{\omega(t-\tau)}{\xi}\right]$

Let $\eta=(B+k J) / J, \Omega=\omega / \xi$ and if the time-delay constant $\tau$ is much larger than $1 / \eta$ (the condition can be satisfied when $k$ is large), an iterative solution (15-16) can be got from (14).

$\omega_{n+1}(t) \approx \xi \mu \sin \left[\frac{\omega_{n}(t)}{\xi}\right]$ 
TABLE III

PARAMETERS OF DC MOTOR

\begin{tabular}{lll}
\hline Parameter & Value & Unit \\
\hline$B$ & 0.003 & $\mathrm{Nms} / \mathrm{rad}$ \\
$J_{m}$ & $1.5 \times 10^{-5}$ & $\mathrm{Kgms}^{2}$ \\
$K_{E}$ & 0.22 & $\mathrm{Vs} / \mathrm{rad}$ \\
$K_{T}$ & $22.5 \times 10^{-3}$ & $\mathrm{kgm} / \mathrm{A}$ \\
$L_{m}$ & 3.4 & $\mathrm{mH}$ \\
\hline
\end{tabular}

$\Omega_{n+1}(t) \approx \mu \sin \left[\Omega_{n}(t)\right]$

The criterion of chaos for this iterative map is the famous period three imply chaos theorem [7]: Let $\mathfrak{R}$ be an interval and map $P: \mathfrak{R} \rightarrow \mathfrak{R}$ be continuous. Assume there is a point $a \in \mathfrak{R}$ for which the points $b=P(a)$, $c=P(b)=P^{2}(a)$ and $d=P^{3}(a)$ satisfy:

$d \leq a<b<c$ (or $d \geq a>b>c)$

Then for every $k=1,2, \cdots$, there is a periodic point in $\mathfrak{R}$ having period $k$.

Reference [8] has verified the existence of points that satisfy condition (17) for fixed sufficiently large $\mu$. For example, $a=0.3$ is such a point for $\mu=3.54$. So the map defined by (16) can be proved to be chaotic with sufficiently large $\mu$.

For experimental verification, a practical DC motor (Sanyo Denki Super L720T) is adopted. Its main parameters are listed in Table III. In the experiment the torque parameter $\mu=100$, the speed parameter $\xi=12$ and the

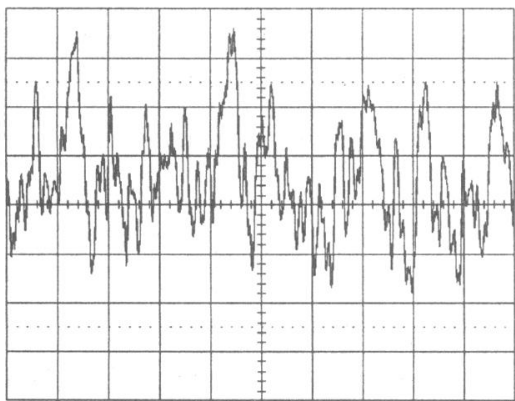

Fig. 3. Measured waveform of motor speed ( $174.5 \mathrm{rad} / \mathrm{s} / \mathrm{div}$, $0.1 \mathrm{~s} / \mathrm{div})$

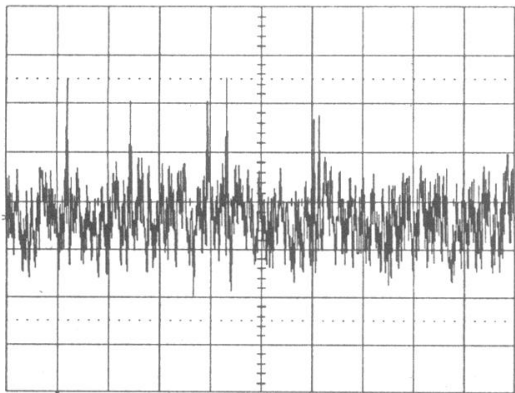

Fig. 4. Measured waveform of armature current (1.35A/div, $0.1 \mathrm{~s} / \mathrm{div})$

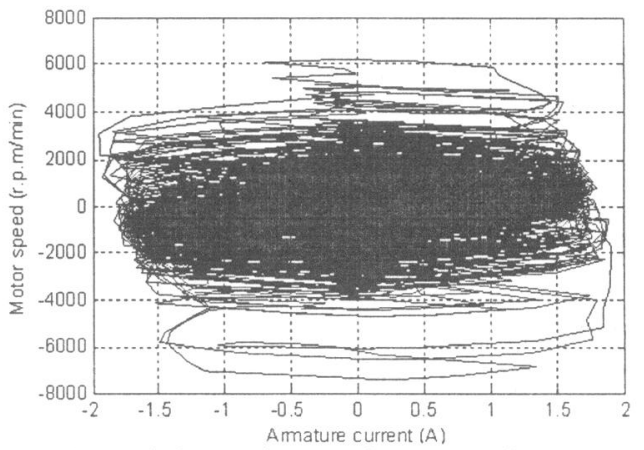

Fig. 5. Measured phase trajectory of motor speed versus armature current.

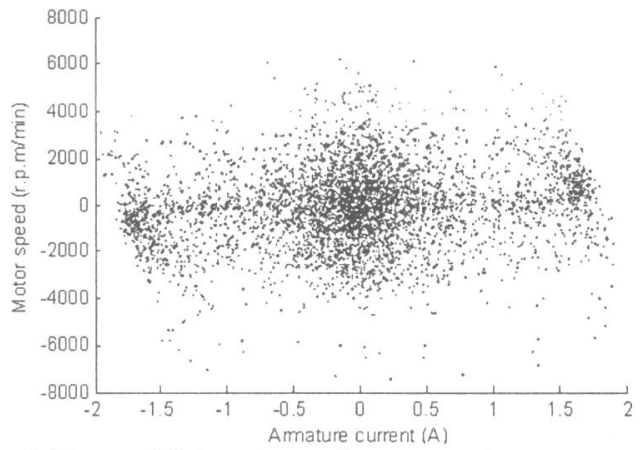

Fig. 6. Measured Poincaré map of motor speed versus armature current.

time-delay parameter $\tau=3 \mathrm{~s}$. Soil parameters is set as soft clay parameters and the sampling time is set as the timedelay parameter. Based on the aforementioned time-delay feedback control system, this DC motor is chaoized for the proposed compactor. Fig. 3 and Fig. 4 show the measured waveforms of motor speed and armature current respectively. The corresponding measured phase portrait and Poincaré map on the plate of motor speed versus armature current are also plotted in Fig. 5 and Fig. 6 respectively. As expected, they exhibit random-like and bounded properties which are well-known characteristics of chaos.

\section{RESULTS}

To describe the advantages of electrically chaotic compactor, the displacement of drum, the vertical exciting force transmitted to surface of soil [1], and the average compaction energy per unit volume [2] are compared between the constant speed compactor and electrically chaotic compactor with the same average output power. Because practical soil is made of different components, the comparison should be carried on with different soil pa-

TABLE IV

CONTROL PARAMETERS

\begin{tabular}{lll}
\hline Parameter & Value & Unit \\
\hline$\mu$ & 100 & \\
$\xi$ & 12 & \\
$\tau$ & 3 & \\
$k$ & 10 & \\
\hline
\end{tabular}


rameters. The final comparison results will be got by considering all soil conditions used for simulation. The rotating speed of constant speed compactor is $70 \mathrm{rad} / \mathrm{s}$ and the parameters of time-delay feedback control law are listed in TABLE IV. In order to simulate the soil composed of different soil type, 50 natural frequencies are chosen averagely between the maximum natural frequency and the minimum natural frequency of soil listed in TABLE II. The total average output power of the constant speed compactor and electrically chaotic compactor are $348 w$ and $342 \mathrm{w}$ respectively.

It can be concluded that compaction effect is better if the displacement of drum and the vertical exciting force transmitted to soil is larger. Considering the motion of electrically chaotic compactor is irregular, the average displacement and the average vertical exciting force transmitted to soil substitute for the peak value of periodic motion. Fig. 7(a) shows the peak displacement of drum in constant speed compactor with 50 groups of different soil parameters. Fig. 7(b) shows the average displacement of drum in electrically chaotic compactor with 50 groups of different soil parameters. The horizontal lines in the figure represent the average value of 50 groups of simulations. The average values for constant speed compactor and electrically chaotic compactor are $0.26 \mathrm{~m}$ and $0.50 \mathrm{~m}$ respectively. Fig. 8(a) shows the peak vertical force transmitted to soil in constant speed compactor with 50 groups of different soil parameters. Fig. 8(b) shows the

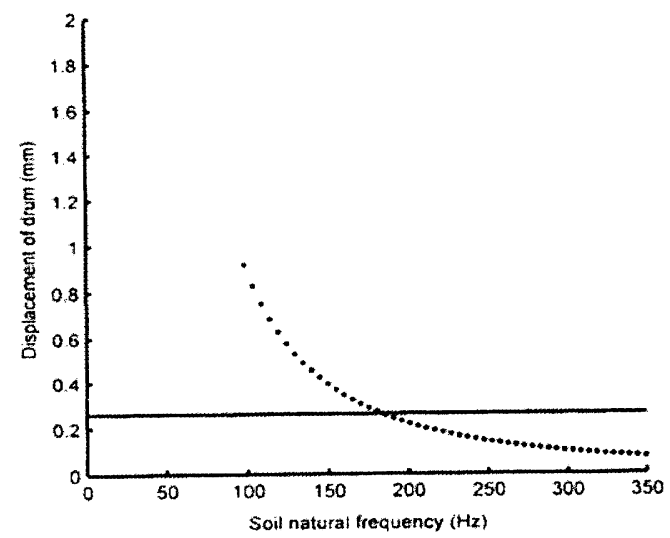

(a)

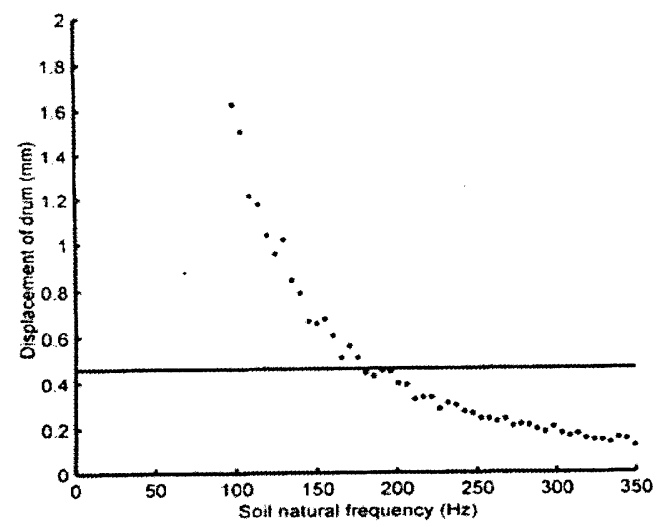

(b)

Fig. 7. Displacement of drum: (a) constant speed compactor; (b) electrically chaotic compactor.

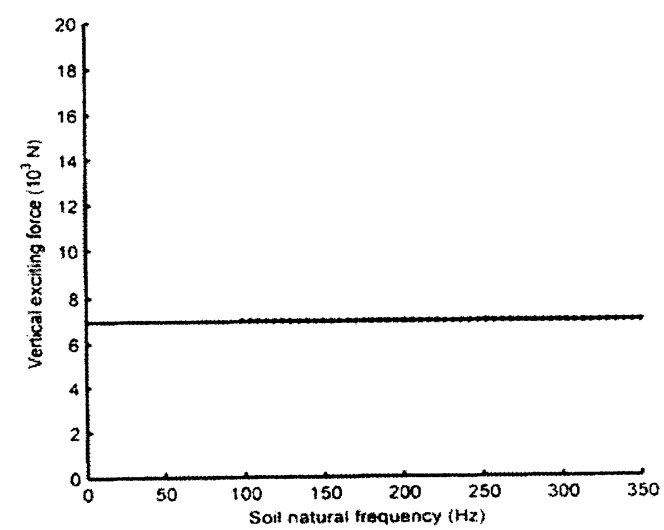

(a)

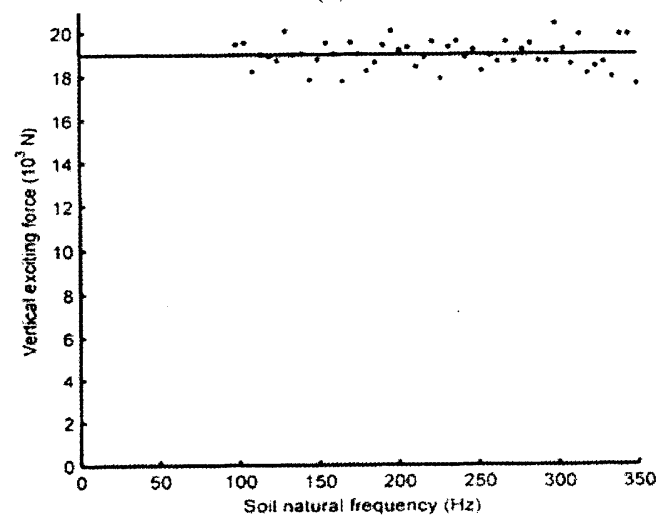

(b)

Fig. 8. Vertical exciting force transmitted to soil: (a) constant speed compactor; (b) electrically chaotic compactor.

peak vertical force transmitted to soil in electrically chaotic compactor with 50 groups of different soil parameters. The horizontal lines in the figure represent the average value of 50 groups of simulations. The average values for constant speed compactor and electrically chaotic compactor are $6.96 \mathrm{kN}$ and $18.96 \mathrm{kN}$ respectively. Not only the displacement of drum but also the vertical exciting force transmitted to soil shows that the electrically chaotic compactor offers better compaction effect than constant speed compactor.

The average compaction energy per unit volume of soil $E$ can be used to evaluate the compaction performance. $E$ can be calculated by (18) [2].

$$
E=2 \beta_{v s}\left(W+\frac{F_{v}}{2}\right) \frac{f N_{T}}{V B_{u} h}
$$

where $\beta_{\mathrm{vs}}$ is the amplitude of displacement of drum; $W$ is the total weight of the compactor; $F_{r}$ is the peak vertical exciting force; $f$ is the frequency of motor; $N_{T}$ is the number of compacting passes; $V$ is the vehicle speed; $B_{w}$ is the width of compaction, and $h$ is the thickness of thick-lift soil. $V, N_{T}, B_{w}$ and $h$ are set with the same values during comparison between the constant speed compactor and the electrically chaotic compactor. $V=0.001 \mathrm{~m} / \mathrm{s}, N_{T}=1, B_{w}=0.2 \mathrm{~m}$ and $h=0.1 \mathrm{~m}$. Because 


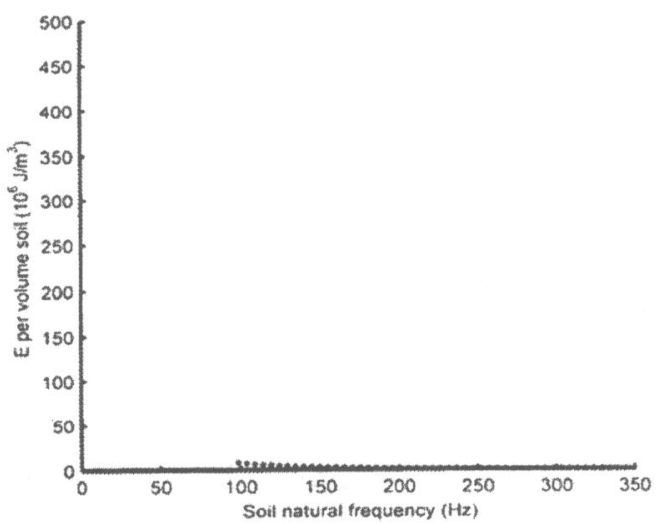

(a)

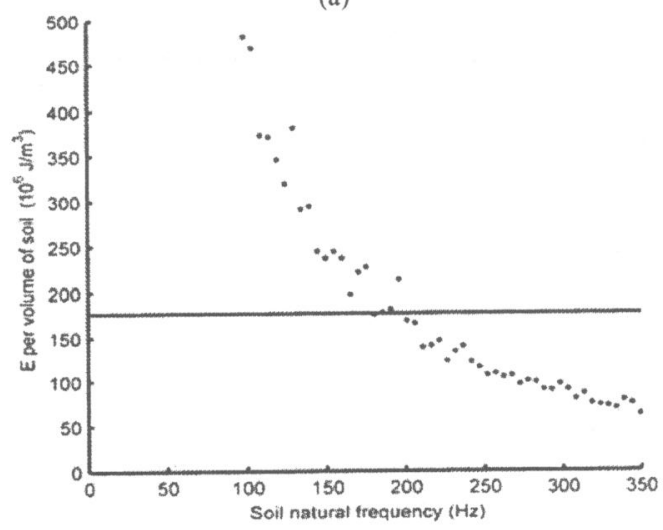

(b)

Fig. 9. Average compaction energy per unit volume of soil: (a) constant speed compactor; (b) electrically chaotic compactor.

the electrically chaotic compactor comprises many vibratory frequencies, the total energy transmitted to soil can be evaluated by accumulating energy at different vibratory frequency. Fig. 9(a) shows the peak $E$ in constant speed compactor with 50 groups of different soil parameters. Fig. 9(b) shows the peak $E$ in electrically chaotic compactor with 50 groups of different soil parameters. The horizontal lines in the figure represent the average value of 50 groups of simulations. The average values of constant speed compactor and electrically chaotic compactor are $2.48 \times 10^{6} \mathrm{~J} / \mathrm{m}^{3}$ and $176.88 \times 10^{6} \mathrm{~J} / \mathrm{m}^{3}$ respectively. It is obvious that the peak $E$ of electrically chaotic compactor is much larger than that of constant speed

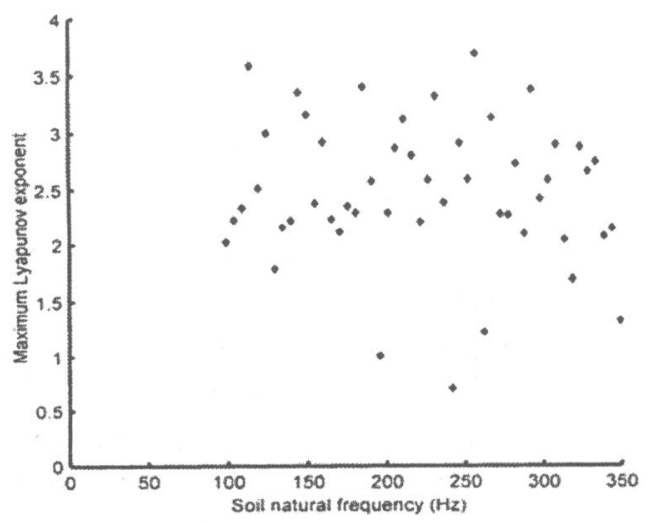

Fig. 10. Corresponding maximum Lyapunov exponent versus soil natural frequency. compactor.

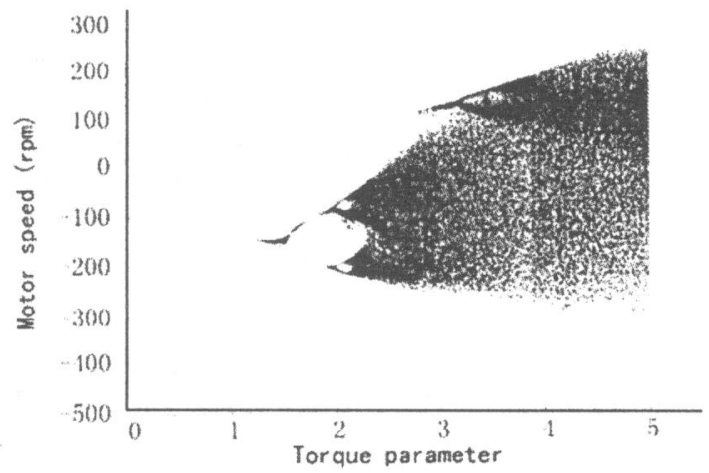

Fig. 11. Motor speed bifurcation diagram via torque parameter

To prove the existence of chaos strictly, Fig. 10 displays the corresponding maximum Lyapunov exponent of each simulation with time series of motor speed [9-10]. It is shown that each maximum Lyapunov exponent is greater than unity, hence mathematically confirming the existence of chaos.

Compared to the mechanically chaotic compactor, the electrically chaotic compactor can offer good controllability and high flexibility. The chaotic feature and structure of chaotic attractor will be fixed once the size parameters of mechanically chaotic compactor are confirmed. On the other hand, the chaotic feature of electrically chaotic compactor can be changed by adjusting the torque parameter $\mu$. Fig. 11 shows the bifurcation diagram of motor speed respect to $\mu$. The bifurcation diagram shows route from the periodic motion to chaotic motion of electrically chaotic compactor with increase of $\mu$. By selecting $\mu=1,4.5$ and 100 , the power density distributions for acceleration of drum are shown in Fig. 12. It can be seen that the power density distribution will become wider with increase of $\mu$. This ability of offering different chaotic motion by adjusting control parameter enables the electrically chaotic compactor to attain good compaction effect under different soil conditions.

\section{CONCLUSION}

In this paper a novel electrically chaotic compactor is proposed and implemented. Different from the mechanically chaotic one, this electrically chaotic compactor will adopt modified time-delay feedback control to chaoize a DC motor which is coupled with only one eccentric mass. The dynamic models of constant speed compactor and electrically chaotic compactor are constructed respectively. The Poincaré map of electrically chaotic compactor is deducted to verify the existence of chaos theoretically and practical experiment proves the chaotic feature in motor. Computer simulation shows that the electrically chaotic compactor has better compaction effect than constant speed compactor. The maximum Lyapunov exponent verifies the existence of chaos in the simulation strictly. The bifurcation diagram and power density distribution show the chaotic motion can be changed by adjust- 


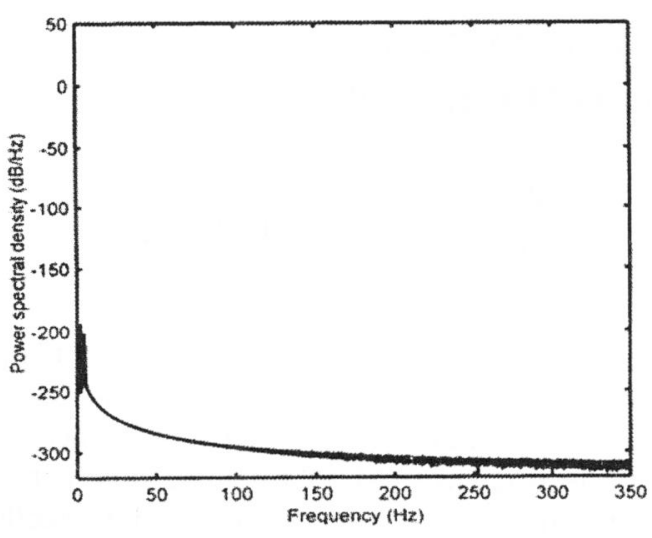

(a)

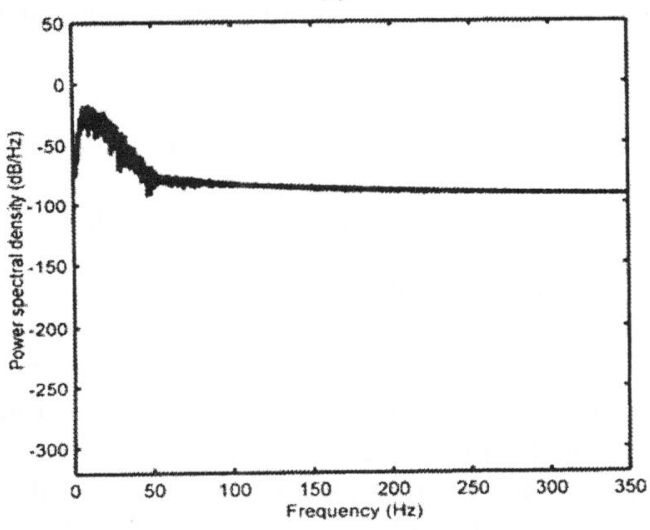

(b)

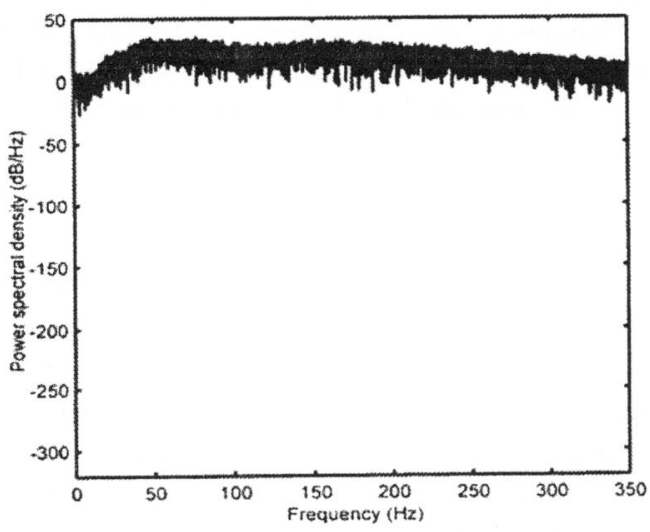

Fig. 12. Power density distribution of drum acceleration: (a) $\mu=1$; (b) $\mu=4.5$; (c) $\mu=100$.

ing control parameter in electrically chaotic compactor. The ability enables the electrically chaotic compactor to offer good controllability and high flexibility rather than the mechanically chaotic compactor.

\section{REFERENCES}

[1] T. S. Yoo, and E. T. Selig, "Dynamics of vibratory-roller compaction," Journal of Geotechnical Engineering Division, pp. 1211-1231, Oct. 1979.

[2] D. T. Tran, and T. Muro, "Effect of an innovate vertical vibrotracked vehicle on soil compaction," Journal of Terramechanics, vol. 41, pp. 1-23, 2004.

[3] Y. J. Long, Y. Yang, and C. L. Wang, "Road roller engineering based on chaotic vibration mechanics," Engineering Science of China, vol. 2, no. 9, pp. 76-79, 2000.
[4] K. T. Chau, S. Ye, and Y. Gao, "Application of chaotic-motion motors to industrial mixing processes," IEEE Industrial Application Society Annual Meeting, pp. 1874-1880, 2004.

[5] Dept. of Defense, USA, "Soil dynamics and special design aspects, $M I L-H D B K-1007 / 3$, pp. 19-28, 1997.

[6] D. A. Brown, M. W. O’Neill, M. Hoit, M. Mcvay, M. H. El Naggar, and S. Chakraborty, "NCHRP Report 461-Static and dynamic lateral loading of pile groups," National Academic Press, pp. B-26, 2001.

[7] T. Y. Li, and J. A. Yorke, "Period three implies chaos," The American Mathematical Monthly 82, pp. 481-485, 1975.

[8] Y. Gao, and K. T. Chau, "Chaotification of permanent-magnet synchronous motor drives using time-delay feedback," IEEE $28^{\text {th }}$ Annual Conference of Industrial Electronics Society, vol. 1, pp. 762-766, Nov. 2002

[9] C. J. Cellucci, A. M. Albano, P. E. Rapp, R. A. Pittenger, and R. C. Josiassen, "Detecting noise in a time series," Chaos, vol. 7, no. 3, pp. 414-422, 1997.

[10]M. T. Rosenstein, J. J. Collins, and C. J. De Luca, "A practical method for calculating largest Lyapunov exponents from small data sets," Physica D, vol. 65, issues. 1-2, pp. 117-134, May. 1993. 